\title{
Lattice Statistics and Dynamics within Cluster Variation Method
}

\author{
Yasunori Yamada ${ }^{1, *}$ and Tetsuo Mohri ${ }^{2}$ \\ ${ }^{1}$ Department of Materials Processing, Graduate School of Engineering, Tohoku University, Sendai 980-8577, Japan \\ ${ }^{2}$ Institute for Materials Research, Tohoku University, Sendai 980-8577, Japan
}

The conventional Cluster Variation Method (CVM) does not explicitly consider both lattice vibration and local lattice relaxation effects. As discussed in the previous studies, these deficiencies result in overestimation of order-disorder transformation temperatures for the $\mathrm{Cu}$ - $\mathrm{Au}$ phase diagram. In the present paper, we propose modified CVM which explicitly takes both the effects mentioned above into account. By employing the present modified CVM, we evaluated both lattice vibration and local lattice relaxation effects, and estimated free energy of mixing for $\mathrm{Cu}-\mathrm{Au}$ disordered phase at a finite temperature. [doi:10.2320/matertrans.MBW201504]

(Received October 14, 2015; Accepted January 20, 2016; Published March 25, 2016)

Keywords: cluster variation method, lattice vibration, local lattice relaxation, free energy of mixing

\section{Introduction}

Cluster Variation Method (CVM) provides a hierarchy structure for approximation of entropy in terms of a basic cluster which is the largest cluster in its formula. Usually, CVM approximations are termed based on their basic clusters. For example, when basic cluster is a pair (tetrahedron) cluster, the approximation is referred to as pair (tetrahedron) approximation. Since the time when Kikuchi ${ }^{1)}$ had proposed Cluster Variation Method (CVM), various applications of CVM have been proposed such as coherent interface ${ }^{2)}$, SRO hardening ${ }^{3)}$, effective pair interaction energy ${ }^{4)}$, diffuse intensity scattering ${ }^{5)}$, phase equilibria and phase diagram ${ }^{6-9)}$, and coupling with $\mathrm{PFM}^{10,11)}$. In particular, a number of studies for phase diagram calculation ${ }^{6-8)}$ have been conducted because CVM yields more reasonable transformation temperature than Brag-Williams approximation as shown in Ref. 12).

However, as discussed in the previous study ${ }^{6}$, the conventional CVM overestimates order-disorder transformation temperatures for $\mathrm{Cu}$-Au system. It is pointed out that one of the causes of this overestimation is the neglect of local lattice relaxation which is a structural relaxation due to mixing of different sized-atoms ${ }^{6,9)}$. Essentially, the conventional CVM is designed for a fixed lattice system where atomic displacements are not allowed. Then, local lattice relaxation, as well as lattice vibration, is not explicitly considered.

In order to incorporate atomic displacements into a CVM formula, Kikuchi et al. ${ }^{13)}$ and Finel ${ }^{14,15)}$ proposed Continuous Displacement Cluster Variation Method (CDCVM) and Gaussian CVM, respectively. However, CDCVM requires an intractable number of variational parameters for three-dimensional calculations. To the best of authors' knowledge, there is no report about a CDCVM for three-dimensional system beyond the pair approximation ${ }^{16)}$ although it is inevitable to employ at least tetrahedron approximation for order-disorder transformation on a fcc system ${ }^{12)}$. While Gaussian CVM has less restriction about the number of variational parameters, it has not been developed for multi-component system yet. Hence, Gaussian CVM considers only vibrational free energy for a single component system, but not local lattice relax-

*Graduate Student, Tohoku University. Corresponding author, E-mail: y.yamada@imr.tohoku.ac.jp ation.

In the present paper, we propose an alternative model of CVM in order to overcome the above problems. The present CVM can consider a local lattice relaxation and vibrational free energy without variational parameters describing atomic distributions around a lattice point, which makes it easy to apply the present model to a three-dimensional system.

By employing the present CVM, we estimate a free energy of mixing of $\mathrm{Cu}-\mathrm{Au}$ disordered phase at finite temperature within tetrahedron approximation. In addition, we evaluate energy contributions of both local lattice relaxation and lattice vibration to the free energy of mixing.

\section{Calculation Model}

We described only an outline of derivation procedure of the present CVM due to the restriction of the number of pages. The details of the derivation procedure will be reported elsewhere.

First, we define a Hamiltonian $H[\mathbf{p}, \mathbf{q}, \sigma]$ as the function of $\mathbf{p}, \mathbf{q}$ and $\boldsymbol{\sigma}$, where $\mathbf{p}$ and $\mathbf{q}$ are vectors representing momentums and positions of atoms, respectively, and $\sigma$ is a configuration of spin variables $\sigma_{n}$ over the lattice points $n$, i.e. $\sigma=$ $\left(\sigma_{1}, \sigma_{2}, \sigma_{3}, \ldots\right)$. A spin variable $\sigma_{n}$ represents an atomic species. Then, the Hamiltonian $H[\mathbf{p}, \mathbf{q}, \boldsymbol{\sigma}]$ is constituted by the kinetic energy $K[\mathbf{p}, \sigma]$ and potential energy $U[\mathbf{q}, \sigma]$,

$$
H[\mathbf{p}, \mathbf{q}, \sigma]=K[\mathbf{p}, \sigma]+U[\mathbf{q}, \sigma] .
$$

Kinetic energy $K[\mathbf{p}, \sigma]$ is given by $K[\mathbf{p}, \sigma]=\sum_{n} \sum_{i=1}^{3} p_{n i}{ }^{2} / 2 M_{\sigma_{n}}$, where $p_{n i}$ is momentum on $n$ - lattice point and $M_{\sigma_{n}}$ is a mass of atomic species represented by $\sigma_{n}$. The index $i$ indicates the coordinate axes of orthogonal system, $i=1,2,3$.

Here, we approximate potential function by the second order Taylor expansion around lattice position $\mathbf{q}^{0}$,

$$
U[\mathbf{q}, \sigma] \approx U^{0}+\mathbf{v}^{t}\left(\mathbf{q}-\mathbf{q}^{0}\right)+\frac{1}{2}\left(\mathbf{q}-\mathbf{q}^{0}\right)^{t} \mathbf{W}\left(\mathbf{q}-\mathbf{q}^{0}\right),
$$

where

$U^{0} \equiv U\left[\mathbf{q}^{0}, \boldsymbol{\sigma}\right], \mathbf{v} \equiv\left(\frac{\partial U\left[\mathbf{q}^{0}, \boldsymbol{\sigma}\right]}{\partial q_{n i}}\right)_{n i}, \mathbf{W} \equiv\left(\frac{\partial^{2} U\left[\mathbf{q}^{0}, \boldsymbol{\sigma}\right]}{\partial q_{n i} \partial q_{m j}}\right)_{n i, m j}$ 
Equation (2) is written in matrix form, where $\mathbf{v}$ is a vector and $\mathbf{W}$ is a matrix. $\mathbf{q}^{0}$ indicates a vector of all lattice positions and $q_{n i}$ is an atomic position of $n$ th- lattice point in $i$ - direction.

It is important to notice that atoms are treated as distinguishable particles associated with lattice position $\mathbf{q}^{0}$ in eq. (2). In this circumstance, all states of the system are described in a continuous real and momentum space and a discrete space spanned by spin variables. Then, we define a partition function $Z$ as

$$
Z=\frac{1}{(2 \pi \hbar)^{3|L|}} \iint \operatorname{Tr}_{\boldsymbol{\sigma}} \exp \left(-\frac{H[\mathbf{p}, \mathbf{q}, \boldsymbol{\sigma}]}{k_{\mathrm{B}} T}\right) \prod_{n \in L} \prod_{i=1}^{3} \mathrm{~d} p_{n i} \mathrm{~d} q_{n i}
$$

where $L$ is a set of all atoms, $|L|$ is the number of all atoms, $h$ is Plank constant and $\hbar=h / 2 \pi$, and $\operatorname{Tr}_{\sigma}$ indicates a configurational sum over $\sigma$. By using Gaussian integral with respect to $\mathbf{p}$ and $\mathbf{q}$, eq. (4) can be reduced to the following equation,

$$
Z=\underset{\sigma}{\operatorname{Tr}} \exp \left(-\frac{U^{0}[\sigma]+U^{\mathrm{r}}[\sigma]+F^{\mathrm{v}}[\sigma]}{k_{\mathrm{B}} T}\right)
$$

where

$$
\begin{gathered}
U^{0}[\boldsymbol{\sigma}]=U\left[\mathbf{q}^{0}, \boldsymbol{\sigma}\right], \\
U^{\mathrm{r}}[\boldsymbol{\sigma}] \equiv-\frac{1}{2} \mathbf{v}^{t} \mathbf{W}^{-1} \mathbf{v}
\end{gathered}
$$

and

$$
F^{\mathrm{v}}[\sigma]=k_{\mathrm{B}} T \sum_{k=1}^{3|L|} \ln \left[\frac{\hbar \omega_{k}}{k_{\mathrm{B}} T}\right]
$$

It is worth pointing out that the functions $U^{0}[\sigma], U^{\mathrm{r}}[\sigma]$ and $F^{\mathrm{v}}[\sigma]$ indicate formation energy for a fixed lattice, local lattice relaxation energy and vibrational free energy, respectively. In eq. (8), $\omega_{k}$ is an angular frequency of $k$ th- harmonic oscillator, and derived from following characteristic equation,

$$
\operatorname{det}\left|\mathbf{D}-\omega_{k}^{2} \mathbf{I}\right|=0,
$$

where $\mathbf{I}$ is an identical matrix and

$$
\mathbf{D}=\left(\frac{1}{\sqrt{M_{\sigma_{n}} M_{\sigma_{m}}}} \frac{\partial^{2} U\left[\mathbf{q}^{0}, \boldsymbol{\sigma}\right]}{\partial q_{n i} \partial q_{m j}}\right)_{n i, m j} .
$$

It is impossible to obtain exact values of eqs. (7) and (8) except for a perfect crystal, because we cannot describe an atomic configuration of an entire lattice in the thermodynamic limit. Then, we approximate eqs. (7) and (8) by using Möbius inversion formula ${ }^{17,18)}$. In the beginning of this approximation, we introduce a Hamiltonian $H_{\alpha}\left[\mathbf{p}_{\alpha}, \mathbf{q}_{\alpha}, \boldsymbol{\sigma}_{\alpha}\right]$ associated with each cluster, where cluster $\alpha$ is defined as a set of lattice points, $\mathbf{p}_{\alpha}$ and $\mathbf{q}_{\alpha}$ are vectors of momentums and positions of $\alpha$-cluster, respectively, and $\sigma_{\alpha}$ is a configuration of spin variables involved in $\alpha$ - cluster. A Hamiltonian $H_{\alpha}\left[\mathbf{p}_{\alpha}\right.$, $\left.\mathbf{q}_{\alpha}, \boldsymbol{\sigma}_{\alpha}\right]$ is given by $H_{\alpha}\left[\mathbf{p}_{\alpha}, \mathbf{q}_{\alpha}, \boldsymbol{\sigma}_{\alpha}\right]=K_{\alpha}\left[\mathbf{p}_{\alpha}, \boldsymbol{\sigma}_{\alpha}\right]+U_{\alpha}\left[\mathbf{q}_{\alpha}, \boldsymbol{\sigma}_{\alpha}\right]$, where $K_{\alpha}\left[\mathbf{p}_{\alpha}, \sigma_{\alpha}\right]$ and $U_{\alpha}\left[\mathbf{q}_{\alpha}, \sigma_{\alpha}\right]$ are kinetic energy and potential energy, respectively, with $K_{\alpha}\left[\mathbf{p}_{\alpha}, \sigma_{\alpha}\right]=$ $\sum_{n \in a} \sum_{i=1}^{3} p_{n i}{ }^{2} / 2 M_{\sigma_{n}}$. The definition of $U_{\alpha}\left[\mathbf{q}_{\alpha}, \boldsymbol{\sigma}_{\alpha}\right]$ is shown in the next section. By replacing $H[\mathbf{p}, \mathbf{q}, \sigma]$ by $H_{\alpha}\left[\mathbf{p}_{\alpha}, \mathbf{q}_{\alpha}, \sigma_{\alpha}\right]$ in eq. (3), we define $U_{\alpha}^{0}\left[\sigma_{\alpha}\right], U_{\alpha}^{\mathrm{r}}\left[\sigma_{\alpha}\right]$ and $F_{\alpha}^{\mathrm{v}}\left[\sigma_{\alpha}\right]$ in a similar manner with eqs. (6), (7) and (8). Here, we approximate the functions $U^{0}[\sigma], U^{\mathrm{r}}[\sigma]$ and $F^{\mathrm{v}}[\sigma]$ by using a truncation of Möbius inversion formula. This scheme is similar to the formulation of conventional CVM by $\mathrm{An}^{19)}$. We define new functions $\overline{u_{\alpha}^{0}}\left[\sigma_{\alpha}\right], \overline{u_{\alpha}^{\mathrm{r}}}\left[\sigma_{\alpha}\right]$ and $\overline{f_{\alpha}^{\mathrm{v}}}\left[\sigma_{\alpha}\right]$ by the following equation,

$$
\overline{x_{\alpha}}=\sum_{\beta \subseteq \alpha}(-1)^{|\alpha|-|\beta|} X_{\beta},
$$

where $\beta$ is sub-set of $\alpha$, namely, sub-cluster of $\alpha$ - cluster. The number of atoms in $\alpha$ - and $\beta$ - clusters are denoted by $|\alpha|$ and $|\beta|$, respectively. In eq. (11), $\overline{x_{\alpha}}$ corresponds to $\overline{u_{\alpha}^{0}}\left[\sigma_{\alpha}\right], \overline{u_{\alpha}^{\mathrm{r}}}\left[\sigma_{\alpha}\right]$ or $\overline{f_{\alpha}^{\mathrm{v}}}\left[\sigma_{\alpha}\right]$, and $X_{\beta}$ corresponds to $U_{\beta}^{0}\left[\sigma_{\beta}\right], U_{\beta}^{\mathrm{r}}\left[\sigma_{\beta}\right]$ or $F_{\beta}^{\mathrm{v}}\left[\sigma_{\beta}\right]$. According to Möbius inversion formula, the function $X$ of an entire system is given by the summation of $\overline{x_{\alpha}}$ over all $\alpha$ clusters,

$$
X=\sum_{\alpha} \overline{x_{\alpha}},
$$

where $X$ corresponds to $U^{0}[\sigma], U^{\mathrm{r}}[\sigma]$ or $F^{\mathrm{v}}[\sigma]$. For a given set $\Gamma$, if $\left|\overline{x_{\alpha}}\right|<\infty$ for $\alpha \in \Gamma$ and $\overline{x_{\alpha}} \approx 0$ for $\alpha \notin \Gamma$, we can approximate eq. (12) by the following equation,

$$
X \approx \sum_{\alpha \in \Gamma} \overline{x_{\alpha}} .
$$

For an approximation of CVM, $\Gamma$ is given by a set of basic clusters and all their sub-clusters. Thereby, the partition function $Z$ in eq. (5) can be approximated by the following equation.

$$
Z \approx \operatorname{Tr}_{\sigma} \exp \left\{-\frac{1}{k_{\mathrm{B}} T} \sum_{\alpha \in \Gamma}\left(\overline{u_{\alpha}^{0}}\left[\sigma_{\alpha}\right]+\overline{u_{\alpha}^{\mathrm{r}}}\left[\sigma_{\alpha}\right]+\overline{f_{\alpha}^{\mathrm{v}}}\left[\sigma_{\alpha}\right]\right)\right\}
$$

The function $\overline{u_{\alpha}^{0}}\left[\sigma_{\alpha}\right], \overline{u_{\alpha}^{\mathrm{r}}}\left[\sigma_{\alpha}\right]$ and $\overline{f_{\alpha}^{\mathrm{v}}}\left[\sigma_{\alpha}\right]$ are invariant under translation. If we consider a thermodynamic limit, a free energy function can be obtained by using variation principle ${ }^{20)}$ as

$$
\begin{aligned}
\frac{F}{|L|} \approx \min _{\{\rho[\sigma]\}} \operatorname{Tr}_{\sigma} \rho[\sigma]\left\{\sum_{0 \in \alpha \in \Gamma} \frac{1}{|\alpha|}\left(\overline{u_{\alpha}^{0}}\left[\sigma_{\alpha}\right]+\overline{u_{\alpha}^{\mathrm{r}}}\left[\sigma_{\alpha}\right]+\overline{f_{\alpha}^{\mathrm{v}}}\left[\sigma_{\alpha}\right]\right)\right. \\
\left.+\frac{k_{\mathrm{B}} T}{|L|} \ln \rho[\sigma]\right\}
\end{aligned}
$$

where $\rho[\sigma]$ is a probability function of $\sigma$ and satisfies a normalization condition, $\mathbf{0}$ represents origin of a lattice and $\min _{\{\rho[\sigma]\}}$ indicate minimization operators with respect to $\{\rho[\sigma]\}$. It should be noted that $\operatorname{Tr}_{\sigma} \rho[\sigma]=1$. By using eqs. (11) and (13), the entropy term $\left(k_{\mathrm{B}} T /|L|\right) \ln \rho[\sigma]$ in eq. (15) is approximated by

$$
\frac{k_{\mathrm{B}} T}{|L|} \ln \rho[\sigma] \approx-T \sum_{0 \in \alpha \in \Gamma} \overline{s_{\alpha}^{c}}\left[\sigma_{\alpha}\right]
$$

where $\overline{s_{\alpha}^{c}}=\sum_{\beta \subseteq \alpha}(-1)^{|\alpha|-|\beta|} S_{\beta}^{c}$ with $S_{\beta}^{c}=-k_{\mathrm{B}} \ln \rho_{\beta}\left[\sigma_{\beta}\right]$. In eq. (16), $\rho_{\beta}\left[\sigma_{\beta}\right]$ indicates a partial probability of $\beta$ - cluster which is defined by

$$
\rho_{\beta}\left[\sigma_{\beta}\right]=\operatorname{Tr}_{\sigma_{L \vee \beta}} \rho[\sigma],
$$


where trace $\operatorname{Tr}_{\sigma_{\alpha \backslash \beta}}$ is taken over all configuration of $\sigma_{\alpha \backslash \beta}$, where $\alpha \bigvee \beta$ is the set which consists of the elements of $\alpha$ excluding the elements of $\beta$, i.e. $\alpha \backslash \beta=\{n \mid n \in \alpha \wedge n \notin \beta\}$. By substituting eqs. (16) and (17) into eq. (15), the free energy function is obtained as

$$
\begin{aligned}
\frac{F}{|L|} \approx & \min _{\{\rho[\sigma]\}} \sum_{0 \in \alpha \in \Gamma} \frac{1}{|\alpha|} \operatorname{Tr}_{\sigma_{\alpha}} \rho_{\alpha}\left[\sigma_{\alpha}\right] \overline{u_{\alpha}^{0}}\left[\sigma_{\alpha}\right]+\overline{u_{\alpha}^{\mathrm{r}}}\left[\sigma_{\alpha}\right] \\
& \left.+\overline{f_{\alpha}^{\mathrm{v}}}\left[\sigma_{\alpha}\right]-T \overline{s_{\alpha}^{c}}\left[\sigma_{\alpha}\right]\right) .
\end{aligned}
$$

For minimization process, we ignore the restriction by eq. (17), but consider normalization condition $\operatorname{Tr}_{\sigma_{\beta}} \rho_{\beta}\left[\sigma_{\beta}\right]=1$ and geometrical condition $\operatorname{Tr}_{\sigma_{\alpha \backslash \beta}} \rho_{\alpha}\left[\sigma_{\alpha}\right]=\rho_{\beta}\left[\sigma_{\beta}\right]$ for $\alpha, \beta \in \Gamma$. Then, we can replace $\min _{\{\rho[\sigma]\}}$ by $\min _{\left\{\rho_{\alpha}\left[\sigma_{\alpha}\right]\right\}_{\alpha \in \Gamma}}$ in eq. (18) under the normalization and geometrical conditions. In most cases, our main interest in thermal properties is under a constant pressure $P$. Then, Gibbs free energy $G$ is often more useful than Helmholtz free energy $F$. By Legendre transformation, Gibbs free energy $G$ is obtained by the following equation,

$$
\begin{aligned}
\frac{G}{|L|} \approx & \min _{\left\{\rho_{\alpha}\left[\sigma_{\alpha}\right]\right\}_{\alpha \in \Gamma}, V} \sum_{0 \in \alpha \in \Gamma} \frac{1}{|\alpha|} \operatorname{Tr}_{\sigma_{\alpha}} \rho_{\alpha}\left[\sigma_{\alpha}\right]\left(\overline{u_{\alpha}^{0}}\left[\sigma_{\alpha}\right]+\overline{u_{\alpha}^{\mathrm{r}}}\left[\sigma_{\alpha}\right]\right. \\
& \left.+\overline{f_{\alpha}^{\mathrm{v}}}\left[\sigma_{\alpha}\right]-T \overline{s_{\alpha}^{c}}\left[\sigma_{\alpha}\right]\right)+\frac{P V}{|L|}
\end{aligned}
$$

where $V$ is a volume of the system. Hence, the free energy function, which explicitly considers both local lattice relaxation and lattice vibrational effects, is derived.

\section{Calculation Condition}

We calculated the free energy of $\mathrm{Cu}$-Au disordered phase on fcc lattice. We employed tetrahedron approximation of CVM. The basic clusters were given by tetrahedron clusters which consist of only first nearest neighbors. A function $X$ per atom was given by $X /|L| \approx 5 X_{1}-6 X_{2}+2 X_{4}$, where $X_{1}, X_{2}$ and $X_{4}$ indicate the function $X_{\alpha}$ of point, pair and tetrahedron clusters, respectively.

Potential energy was given by a pair approximaiton within the first nearest neighbors. The pair interaction energy was described by the empirical Lennard-Jones potential as

$$
\phi_{\sigma \sigma^{\prime}}[r]=\frac{e_{\sigma \sigma^{\prime}}^{0}}{\kappa_{\sigma \sigma^{\prime}}-\lambda_{\sigma \sigma^{\prime}}}\left\{\lambda_{\sigma \sigma^{\prime}}\left(\frac{r_{\sigma \sigma^{\prime}}^{0}}{r}\right)^{\kappa_{\sigma \sigma^{\prime}}}-\kappa_{\sigma \sigma^{\prime}}\left(\frac{r_{\sigma \sigma^{\prime}}^{0}}{r}\right)^{\lambda_{\sigma \sigma^{\prime}}}\right\},
$$

where $\sigma$ and $\sigma^{\prime}$ denote spin variables, $r$ the interatomic distance, and $e_{\sigma \sigma^{\prime}}^{0}, r_{\sigma \sigma^{\prime}}^{0}, \kappa_{\sigma \sigma^{\prime}}$ and $\lambda_{\sigma \sigma^{\prime}}$ are potential energy parameter whose values employed for the present study are shown in Table 1. We determined these parameters by fitting with cohesive energy, lattice constant and bulk modulus of $\mathrm{Cu}$, $\mathrm{Cu}_{3} \mathrm{Au}, \mathrm{CuAu}, \mathrm{CuAu}_{3}$ and $\mathrm{Au}$, as shown in Table 2. The resultant Lennard-Jones potential is plotted in Fig. 1.

The potential energy $U_{\alpha}\left[\mathbf{q}_{\alpha}, \sigma_{\alpha}\right]$ of $\alpha$ - cluster was defined by the following equation,

$$
\begin{aligned}
U_{\alpha}\left[\mathbf{q}_{\alpha}, \sigma_{\alpha}\right]= & \sum_{n \in \alpha} \sum_{\substack{m \in \alpha \\
m>n}} \phi_{\sigma_{n} \sigma_{m}}\left[r_{n m}\right] \\
& +\sum_{n \in \alpha} \sum_{\substack{m \notin \alpha \\
\{, m\} \text { is first } \\
\text { nerest neighbor }}}^{*}\left\langle\phi_{\sigma_{n} \sigma_{m}}^{0}\left[\mathbf{q}_{\{n\}}\right]\right\rangle,
\end{aligned}
$$

\begin{tabular}{|c|c|c|c|c|}
\hline & & $\begin{array}{c}\text { Lattice } \\
\text { constant } \\
a / \AA\end{array}$ & $\begin{array}{c}\text { Cohesive } \\
\text { energy } \\
E_{\mathrm{c}} / \mathrm{eV}\end{array}$ & $\begin{array}{c}\text { Bulk } \\
\text { modulus } \\
\text { B / GPa }\end{array}$ \\
\hline \multirow[t]{2}{*}{$\mathrm{Cu}(\mathrm{fcc})$} & cal. & 3.61 & 3.487 & 137.2 \\
\hline & (exp.) & (3.62) & $(3.49)$ & (138) \\
\hline \multirow[t]{2}{*}{$\mathrm{Cu}_{3} \mathrm{Au}\left(\mathrm{L1}_{2}\right)$} & cal. & 3.751 & 3.643 & 150.4 \\
\hline & (exp.) & (3.74) & (3.64) & (148) \\
\hline \multirow[t]{2}{*}{$\mathrm{CuAu}\left(\mathrm{L}_{0}\right)$} & cal. & $\begin{array}{l}a=3.968 \\
c=3.674\end{array}$ & 3.747 & 161.8 \\
\hline & (exp.) & $\begin{array}{l}(a=3.966) \\
(c=3.673)\end{array}$ & (3.74) & (163) \\
\hline \multirow[t]{2}{*}{$\mathrm{CuAu}_{3}\left(\mathrm{L1}_{2}\right)$} & cal. & 3.983 & 3.779 & 168.9 \\
\hline & (exp.) & (3.98) & (3.79) & (170) \\
\hline \multirow[t]{2}{*}{$\mathrm{Au}(\mathrm{fcc})$} & cal. & 4.072 & 3.814 & 171.9 \\
\hline & (exp.) & (4.08) & $(3.81)$ & (171) \\
\hline
\end{tabular}

Table 1 Potential energy parameters of Lennard-Jones potential.

\begin{tabular}{|c|c|c|c|}
\hline$\left(\sigma, \sigma^{\prime}\right)$ & $(\mathrm{Cu}, \mathrm{Cu})$ & $(\mathrm{Cu}, \mathrm{Au}),(\mathrm{Au}, \mathrm{Cu})$ & $(\mathrm{Au}, \mathrm{Au})$ \\
\hline$e_{\sigma \sigma^{\prime}}^{0}[\mathrm{eV}]$ & 0.581174 & 0.647235 & 0.635631 \\
\hline$r_{\sigma \sigma^{\prime}}^{0}[\AA]$ & 2.552392 & 2.703572 & 2.879656 \\
\hline$\kappa_{\sigma \sigma^{\prime}}^{0}$ & 7.615058 & 7.313971 & 9.949710 \\
\hline$\lambda_{\sigma \sigma^{\prime}}^{0}$ & 3.413229 & 4.650768 & 4.297272 \\
\hline
\end{tabular}

Table 2 Lattice constant, cohesive energy and bulk modulus. "cal." and "exp." indicate the calculated results by the present Lennard-Jones potential and experimental data ${ }^{9)}$, respectively.

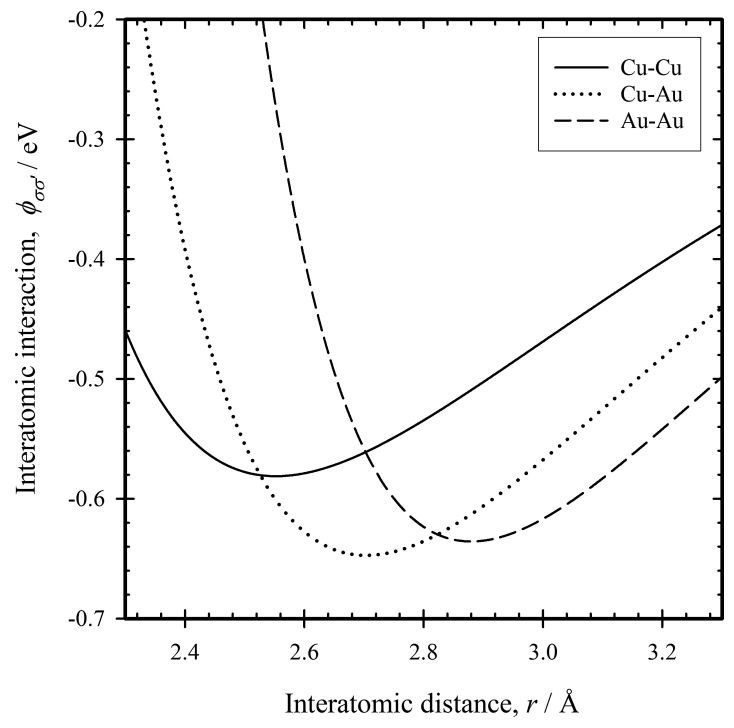

Fig. 1 Lennard-Jones potential. 
where $r_{n m}$ is interatomic distance between atoms $n$ and $m$, i.e. $r_{n m}=\left\|\mathbf{q}_{\{n\}}-\mathbf{q}_{\{m\}}\right\|$, and $\left\langle\phi_{\sigma_{n} \sigma_{m}}^{0}\left[\mathbf{q}_{\{n\}}\right]\right\rangle$ is a mean interaction energy. We define $\left\langle\phi_{\sigma_{n} \sigma_{m}}^{0}\left[\mathbf{q}_{\{n\}}\right]\right\rangle$ as

$$
\left\langle\phi_{\sigma_{n} \sigma_{m}}^{0}\left[\mathbf{q}_{\{n\}}\right]\right\rangle=\sum_{\sigma_{m}} \frac{\rho_{2}\left[\left(\sigma_{n}, \sigma_{m}\right)\right]}{\rho_{1}\left[\left(\sigma_{n}\right)\right]} \phi_{\sigma_{n} \sigma_{m}}\left[r_{n m}^{\prime}\right] .
$$

where $r_{n m}^{\prime}=\left\|\mathbf{q}_{\{n\}}-\mathbf{q}_{\{m\}}^{0}\right\|$, and $\rho_{1}\left[\left(\sigma_{n}\right)\right]$ and $\rho_{2}\left[\left(\sigma_{n}, \sigma_{m}\right)\right]$ denote the partial probability of point and pair cluster. In eq. (21), the summation of the first term is taken over all pair clusters in $\alpha$-cluster, and the summation of the second term is taken over all the first nearest neighbors between the atoms inside and outside $\alpha$-cluster. It is important to note that a potential energy $U_{\alpha}\left[\mathbf{q}_{\alpha}, \sigma_{\alpha}\right]$ defined by eq. (21) satisfies the following equation,

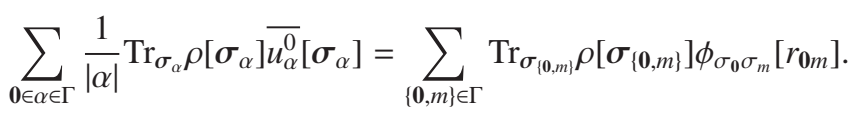

Then, the formation energy for a fixed lattice is given by the summation of interatomic potentials. We note that this is akin to the description of energy by the conventional CVM.

Here, the potential function $U_{\alpha}\left[\mathbf{q}_{\alpha}, \sigma_{\alpha}\right]$ is well-defined, and the Gibbs free energy is given by eq. (19). We also evaluated enthalpy and entropy by $H /|L| \approx$ $\sum_{\mathbf{0} \in \alpha \in \Gamma}(1 /|\alpha|) \operatorname{Tr}_{\boldsymbol{\sigma}_{\alpha}} \rho_{\alpha}\left[\sigma_{\alpha}\right]\left(\overline{u_{\alpha}^{0}}\left[\sigma_{\alpha}\right]+\overline{u_{\alpha}^{\mathrm{r}}}\left[\sigma_{\alpha}\right]\right)+3 k_{\mathrm{B}} T+P V /|L|$ and $S=(H-G) / T$, respectively. The volume $V$ of the system is given as the function of lattice constant $a$, i.e. $V=|L| a^{3} / 4$. The pressure is assigned as $P=0$. The atomic mass of $\mathrm{Cu}$ and $\mathrm{Au}$ are given by 63.55 and $197.0(\mathrm{~g} / \mathrm{mol})$, respectively.

\section{Results and Discussion}

In the present paper, a configurational entropy, local lattice relaxation energy and vibrational free energy are approximated by eqs. (11) and (13). The approximated function of configurational entropy is equivalent to that of the conventional CVM, while the approximation of both local lattice relaxation energy and vibrational free energy by eqs. (11) and (13) have not been attempted. Then, we need to discuss the validity of these approximations, i.e. $F^{\mathrm{v}}[\sigma] \approx \sum_{\alpha \in \Gamma} \overline{f_{\alpha}^{\mathrm{v}}}\left[\sigma_{\alpha}\right]$ and $U^{\mathrm{r}}[\sigma] \approx \sum_{\alpha \in \Gamma} \overline{u_{\alpha}^{\mathrm{r}}}\left[\sigma_{\alpha}\right]$.

First, in order to examine the validity of the approximation of vibrational free energy, we calculated a standard free energy of a pure $\mathrm{Cu}$ and $\mathrm{Au}$. For a single component system, a free energy is given by the summation of formation energy and vibrational free energy. An equilibrium state is given by minimizing eq. (19) with respect to lattice constant $a$ under $\overline{u_{\alpha}^{\mathrm{r}}}[\sigma]$, $\overline{s_{\alpha}^{\mathrm{c}}}[\sigma]=0$.

For comparison, we obtained an analytical solution of the vibrational free energy $F_{\mathrm{AS}}^{\mathrm{v}}$ within quasi-harmonic approximation in classical statistics. By using Fourier transform of position q, eqs. (8) and (9) can be solved by the following equation,

$$
F_{\mathrm{AS}}^{\mathrm{v}}=k_{\mathrm{B}} T \sum_{\mathbf{k}} \sum_{l=1}^{3} \ln \left[\frac{\hbar \omega_{l}[\mathbf{k}]}{k_{\mathrm{B}} T}\right]
$$

coupled with det $\left|\mathbf{D}_{\mathrm{dy}}[\mathbf{k}]-\omega_{l}[\mathbf{k}]^{2} \mathbf{I}\right|=0$, where $\mathbf{D}_{\mathrm{dy}}[\mathbf{k}]$ is 3 by 3 matrix which is given by $\mathbf{D}_{\mathrm{dy}}[\mathbf{k}]=$ $\left((1 / M) \sum_{m}\left(\partial^{2} U / \partial q_{0 i} \partial q_{m j}\right) e^{-i \mathbf{k}^{t}\left(\mathbf{q}_{\{0\}}-\mathbf{q}_{\{m\}}\right)}\right)_{i, j} . \mathbf{k}$ is a wave vector and the summation in eq. (24) is taken over all wave vectors $\mathbf{k}$ in the first Brillouin zone. Then, a free energy of the analytical solution is given by $G^{\mathrm{AS}}=$ $\min _{\mathrm{V}}\left(\sum_{n} \sum_{m<n} \phi_{\sigma_{n} \sigma_{m}}+F_{\mathrm{AS}}^{\mathrm{v}}+P V\right)$. In order to solve the above equations numerically, we used $16 \times 16 \times 16$ sampling points.

Figures 2 (a) and (b) show the temperature dependency of the standard free energy $G^{\text {st }}$, where $G^{\text {st }}=G[T]-G[298.15 \mathrm{~K}]$ for $\mathrm{Cu}$ and $\mathrm{Au}$, respectively. In these figures, the solid line indicates calculated result by the present CVM, the open circles indicate the analytical solutions and the solid circles show experimental data ${ }^{21,22)}$. The calculated results by CVM are consistent with the ones by the analytical solution. This agreement justifies the present approximation of vibrational free energy by eqs. (11) and (13), i.e. $F^{\mathrm{v}} \approx \sum_{\alpha \in \Gamma} \overline{f_{\alpha}^{\mathrm{v}}}$. On the other hand, both the calculated results deviate from experimental data. The reason for this deviation is interpreted as follows. The vibrational free energy depends on elastic properties. However, for the pair approximation of potential energy, the elastic stiffness is restricted by the Cauchy relation $C_{12}=C_{44}$. In addition, for the first nearest neighbor approximation, the elastic stiffness is restricted by the relation $C_{12}=$ $1 / 2 C_{11}$ because of the symmetry of fcc. Then, it is difficult to reproduce elastic properties of $\mathrm{Cu}$ and $\mathrm{Au}$ by using a first nearest neighbor approximation.

Next, we examined the validity of the approximation of local lattice relaxation through eqs. (11) and (13), i.e. $U^{\mathrm{r}} \approx$ $\sum_{\alpha \in \Gamma} \overline{u_{\alpha}^{\mathrm{r}}}$. We calculated enthalpy of mixing for a random phase at $0 \mathrm{~K}$. In this condition, vibrational free energy and configurational entropy become zero, i.e. $\overline{f_{\alpha}^{\mathrm{v}}}=0$ and $\overline{s_{\alpha}^{\mathrm{c}}}=0$ in eq. (19). For a random phase, partial probabilities of pair and tetrahedron cluster are given by a product of partial probabilities of their lattice points. The enthalpy of the mixing is defiend by $\Delta H_{\text {mix }}=H-\rho_{1}[(\mathrm{Cu})] H_{\mathrm{Cu}}-\rho_{1}[(\mathrm{Au})] H_{\mathrm{Au}}$, where $H_{\mathrm{Cu}}$ and $H_{\mathrm{Au}}$ are cohesive energy of pure $\mathrm{Cu}$ and $\mathrm{Au}$, respectively, and $H$ is enthalpy at a stable state. The stable state was obtained by minimizing the enthalpy with respect to a lattice constant $a$.

For the comparison, we performed a simple atomistic calculation with $\mathrm{SQS}^{23)}$. We made nine SQSs which consisted of $10 \times 10 \times 10$ multiples of fcc cells and contained 4000 atoms. The concentration of each SQS is from $\mathrm{Au}-10 \% \mathrm{Cu}$ to $\mathrm{Au}-$ $90 \% \mathrm{Cu}$. Table 3 shows short-range order parameters of the nine SQSs. The total enthalpy is given by $H_{\mathrm{SQS}}[\mathbf{q}, a]=$ $\sum_{n} \sum_{m<n} \phi_{\sigma n} \sigma_{m}\left[r_{n m}\right]+P V$, where $r_{n m}=\left\|\mathbf{q}_{n}-\mathbf{q}_{m}\right\|$. A stable position $\mathbf{q}^{\mathrm{s}}$ and stable lattice constant $a^{\mathrm{s}}$ are determined by minimization of $H_{\mathrm{SQS}}$ with respect to $\mathbf{q}$ and $a$. Local lattice relaxation energy $U_{\mathrm{SQS}}^{\mathrm{r}}$ is defined by $U_{\mathrm{SQS}}^{\mathrm{r}}=H_{\mathrm{SQS}}\left[\mathbf{q}^{0}, a^{\mathrm{s}}\right]-$ $H_{\mathrm{SQS}}\left[\mathbf{q}^{\mathrm{s}}, a^{\mathrm{s}}\right]$.

Figures 3 (a) and (b) show the local lattice relaxation energy and enthalpy of mixing per atom, respectively. For both 
(a) $\mathrm{Cu}$

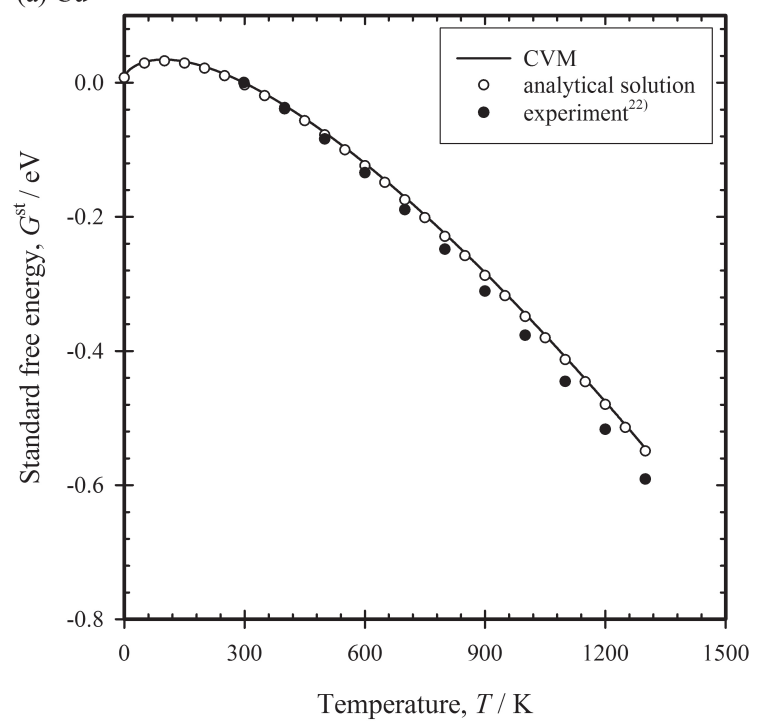

(b) $\mathrm{Au}$

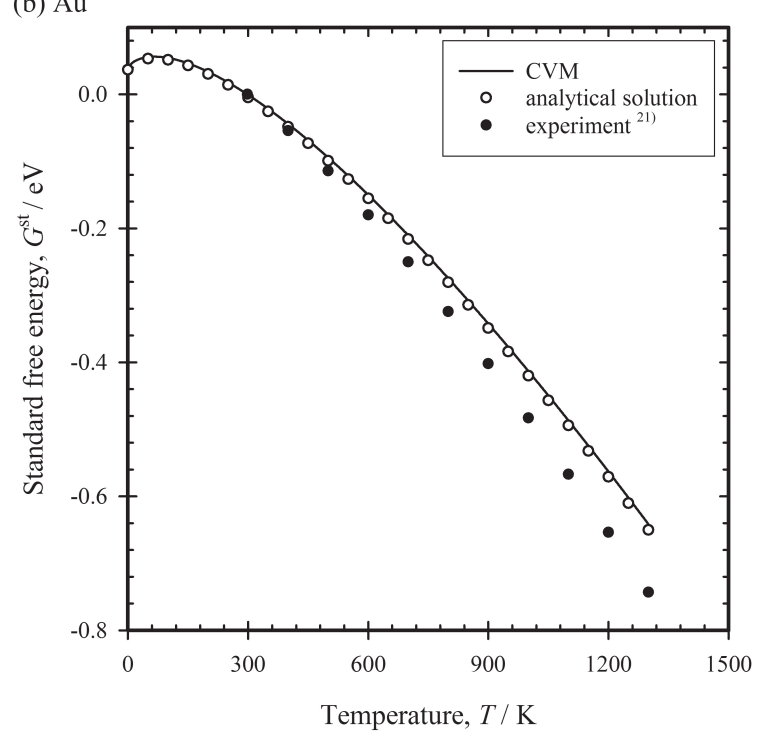

Fig. 2 Standard free energy of (a) $\mathrm{Cu}$ and (b) Au.

Table 3 Short-range order parameters of SQSs. The short-range order parameters $\eta_{i}$ of $i$-th nearest neighbor pair are defined by Warren-Cowley short-range orderparameter, i.e. $\eta_{i}=1-\rho_{2}^{i}[(\mathrm{Cu}, \mathrm{Au})] /\left(\rho_{1}[(\mathrm{Au})] \rho_{1}[(\mathrm{Cu})]\right)$, where $\rho_{2}^{i}[(\mathrm{Cu}, \mathrm{Au})]$ is partial probability of $i$ th- nearest neighbor pair.

\begin{tabular}{cccccc}
\hline at $\% \mathrm{Cu}$ & $a_{1}$ & $a_{2}$ & $a_{3}$ & $a_{4}$ & $a_{5}$ \\
\hline 10 & 0.0000 & 0.0000 & 0.0000 & 0.0037 & -0.0026 \\
20 & 0.0000 & 0.0000 & 0.0000 & -0.0044 & -0.0023 \\
30 & 0.0000 & 0.0000 & -0.0001 & 0.0036 & -0.0020 \\
40 & 0.0000 & 0.0000 & 0.0000 & 0.0082 & -0.0025 \\
50 & 0.0000 & 0.0000 & 0.0000 & 0.0005 & -0.0005 \\
60 & 0.0000 & 0.0000 & 0.0000 & 0.0082 & -0.0025 \\
70 & 0.0000 & 0.0000 & -0.0001 & 0.0036 & -0.0020 \\
80 & 0.0000 & 0.0000 & 0.0000 & -0.0044 & -0.0023 \\
90 & 0.0000 & 0.0000 & 0.0000 & 0.0037 & -0.0026 \\
\hline
\end{tabular}

the results, the present CVM is in reasonable agreement with SQS. In the present CVM, anharmonicity of Lennard-Jones potential is not considered for a given lattice constant because (a) Local relaxation energy

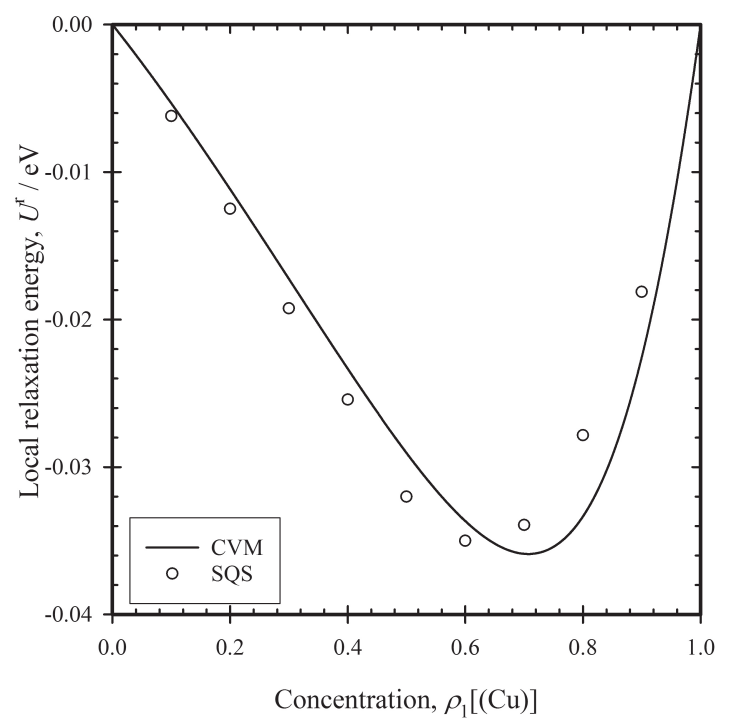

(b)Enthalpy of mixing

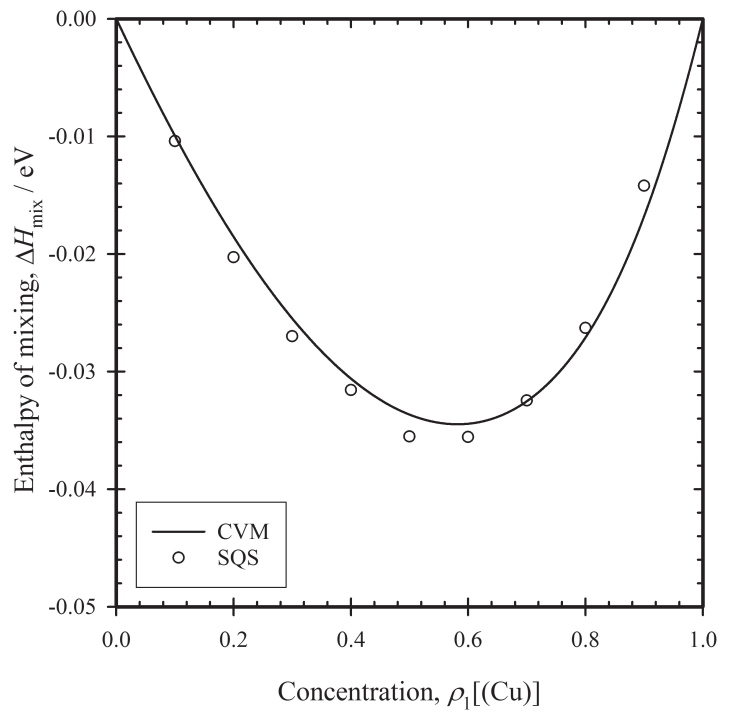

Fig. 3 (a) local lattice relaxation and (b) enthalpy of mixing for random phase of $\mathrm{Cu}-\mathrm{Au}$ system at $0 \mathrm{~K}$.

of second order Taylor expansion of potential energy by eq. (2). In addition, we employed the approximation by eqs. (11) and (13), i.e. $U^{\mathrm{r}} \approx \sum_{\alpha \in \Gamma} \overline{u_{\alpha}^{\mathrm{r}}}$. On the other hand, the calculation with SQS are more reasonable because it does not employ these two approximations. Then, the agreement between CVM and SQS justifies the second order Taylor expansion and the approximation $U^{\mathrm{r}} \approx \sum_{\alpha \in \Gamma} \overline{u_{\alpha}^{\mathrm{r}}}$ for the present interatomic potential function.

Therefore, we conclude that both the approximations of vibrational free energy and local lattice relaxation, $F^{\mathrm{v}} \approx \sum_{\alpha \in \Gamma} \overline{f_{\alpha}^{\mathrm{v}}}$ and $U^{\mathrm{r}} \approx \sum_{\alpha \in \Gamma} \overline{u_{\alpha}^{\mathrm{r}}}$, are rationalized.

$\mathrm{Next}$, we calculated $\mathrm{Cu}-\mathrm{Au}$ disordered phase at $800 \mathrm{~K}$. In this calculation, the formation energy, configurational entropy, local lattice relaxation energy and vibrational free energy are considered. An equilibrium state is given by minimization of eq. (19) with respect to lattice constant $a$ and partial prob-

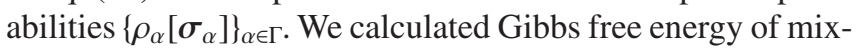
ing $\Delta G_{\text {mix }}$ which is defined by $\Delta G_{\text {mix }}=G-\rho_{1}[(\mathrm{Cu})] G_{\mathrm{Cu}}-$ $\rho_{1}[(\mathrm{Au})] G_{\mathrm{Au}}$, where $G$ is a Gibbs free energy at an equilibrium state, and $G_{\mathrm{Cu}}$ and $G_{\mathrm{Au}}$ are the Gibbs free energy of a pure $\mathrm{Cu}$ 
and Au which were shown in Fig. 2.

Figure 4 shows the concentration dependency of the $\Delta G_{\text {mix }}$, $\Delta H_{\text {mix }}$ and $S_{\text {mix }}=\left(\Delta H_{\text {mix }}-\Delta G_{\text {mix }}\right) / T$. In this figure, three lines indicate the calculated results by CVM, and the symbols $(\bigcirc$, and $\mathbf{\Delta}$ ) indicate experimental data ${ }^{24)}$. The calculated results are in good agreement with the experimental data.

Shown in Fig. 5 are the estimated contributions of formation energy for a fixed lattice $U^{0}$, local lattice relaxation energy $U^{\mathrm{r}}$, vibrational free energy $F^{\mathrm{v}}$ and configurational entropy $-T S^{\mathrm{c}}$ to the free energy of mixing $\Delta G_{\text {mix }}$. It is one of the advantages of the present CVM that free energy function can be separated into each energy term, i.e. $U^{0}, U^{\mathrm{r}}, F^{\mathrm{v}}$ and $-T S^{\mathrm{c}}$.

In Fig. 5, one can see that the major contributions to $\Delta G_{\text {mix }}$ are the formation energy for a fixed lattice $U^{0}$ and configurational entropy $-T S^{\mathrm{c}}$. However, the contribution of a vibrational free energy to the free energy of mixing is small although the temperature dependences of free energies for pure constituents shown in Figs. 2 (a) and (b) are by no means realized without vibrational free energy. Besides, it is noted that it is quite a difficult task to separate the vibrational effect from other terms. A lattice vibration leads to an expansion of lattice constant and a change of potential energy $U\left[\mathbf{q}^{0}, \sigma\right]$. Then, it causes the change of values of formation energy $U^{0}$

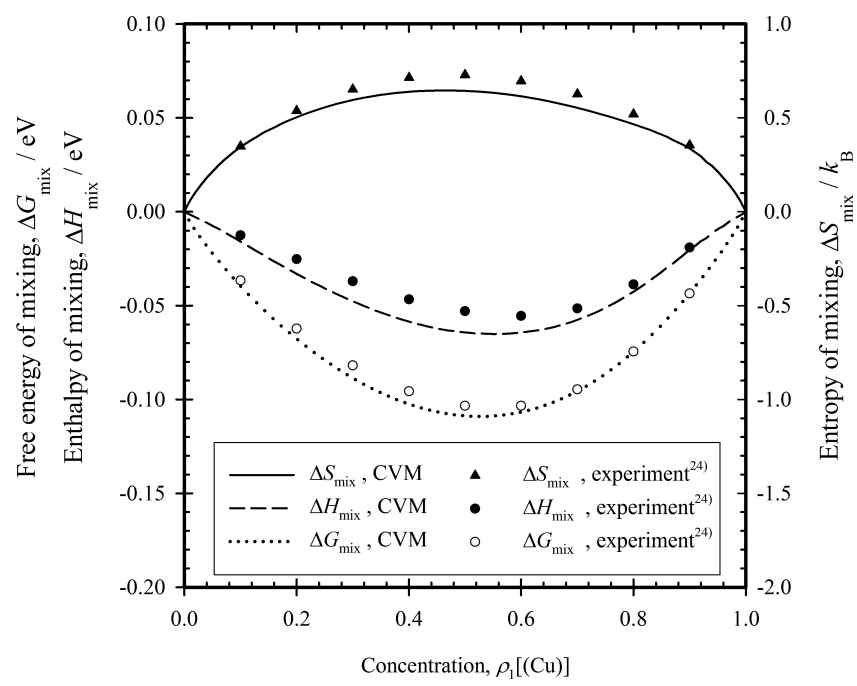

Fig. 4 Gibbs free energy $\Delta G_{\text {mix }}$, enthalpy $\Delta H_{\text {mix }}$ and entropy $\Delta S_{\text {mix }}$ of mixing for $\mathrm{Cu}-\mathrm{Au}$ system at $800 \mathrm{~K}$.

(a) without local lattice relaxation

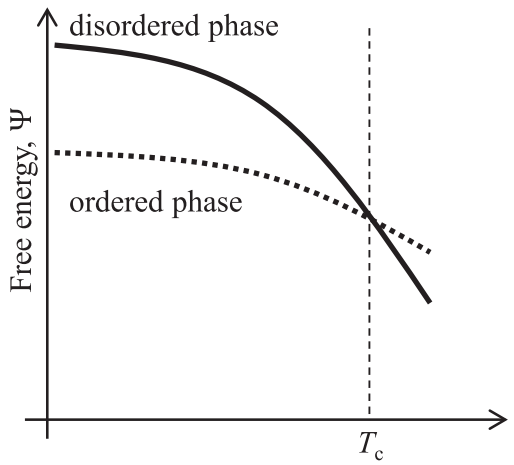

Temperature, $T$ and local lattice relaxation energy $U^{\mathrm{r}}$. On the other hand, the contribution of local lattice relaxation energy $U^{\mathrm{r}}$ is negative and not small. This fact is one of the reasons for the overestimation of transformation temperature by the conventional $\mathrm{CVM}^{6}$ ) which does not consider local lattice relaxation as was mentioned in the Introduction. Local lattice relaxation is caused by mixing of atoms of different size. For $\mathrm{Cu}$-Au disordered phase, local lattice relaxation energy is not small as shown in Fig. 5 because the difference of atomic radius between $\mathrm{Cu}$ and $\mathrm{Au}$ is fairly large, which is larger than $12 \%$. While, when long- and short-range order parameters become higher, a local lattice relaxation energy should become smaller, and then it reaches zero for a perfect ordered phase. For $\mathrm{Cu}$-Au system, order-disorder transformations, i.e. $\mathrm{L}_{2}$-disorder and $\mathrm{L} 1_{0^{-}}$-disorder transformation, are first order transformation. Then, long- or short-range order parameters change drastically before and after the transformation. These facts indicate that the local lattice relaxation energy of disordered phase is negative and much larger than that of ordered phase. The transformation temperature depends on a difference of free energy $\Psi$ between ordered and disordered phase for a given chemical potential $\mu_{\sigma}$, where $\Psi=G-\sum_{\sigma} \mu_{\sigma} N_{\sigma}$ and $N_{\sigma}$

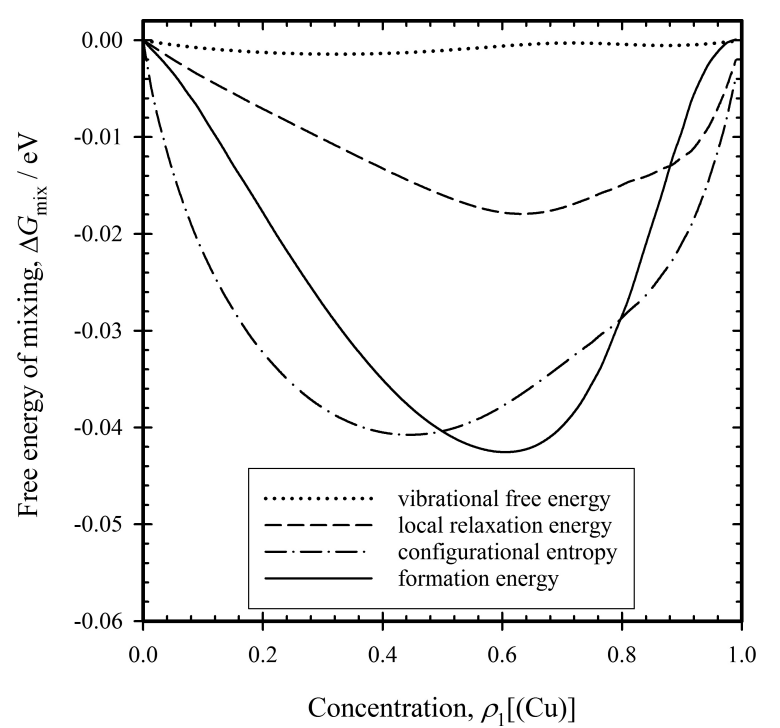

Fig. 5 Free energy contribution of each energy term to Gibbs free energy of mixing for $\mathrm{Cu}-\mathrm{Au}$ system at $800 \mathrm{~K}$.

(b) with local lattice relaxation

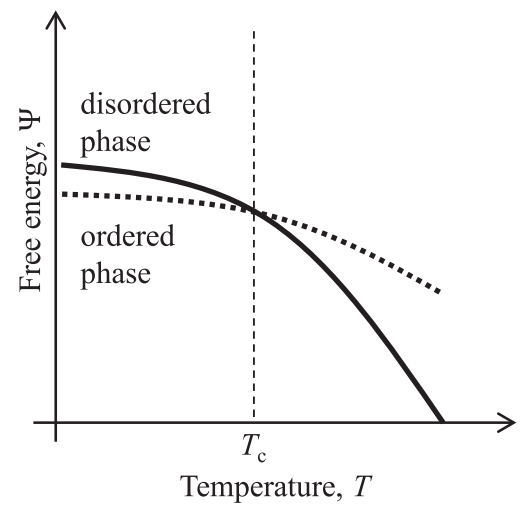

Fig. 6 Relation between order-disorder transformation temperature $T_{\mathrm{c}}$ and free energy curves (a) with local lattice relaxation and (b) without local lattice relaxation. 
Table 4 Comparision of features between the conventional CVM, CDCVM, Gaussian CVM and the present CVM. The symbols without annotation mean as follows. $\times$ : not consider, $\bigcirc$ : consider. ${ }^{* 1}$ real space is divited by quasi-lattice poins, and atomic distribution is approximated as denses of those quasi-lattice poins. ${ }^{* 2}$ atomic distribution is given by Gaussian distribution in continuous real space. ${ }^{* 3}$ Gaussian CVM has not been extended to multi component system. ${ }^{* 4}$ free energy function is derived from partition function in continuous real space.)

\begin{tabular}{lcccc}
\hline & $\begin{array}{c}\text { conventional } \\
\text { CVM }\end{array}$ & CDCVM & $\begin{array}{c}\text { Gaussian } \\
\text { CVM }\end{array}$ & $\begin{array}{c}\text { Present } \\
\text { CVM }\end{array}$ \\
\hline $\begin{array}{l}\text { Formularization in } \\
\text { continuous real space }\end{array}$ & $\times$ & $\triangle^{* 1}$ & $\bigcirc^{* 2}$ & $\bigcirc^{* 4}$ \\
$\begin{array}{l}\text { Local lattice } \\
\text { relaxation }\end{array}$ & $\times$ & $\bigcirc$ & $\times^{* 3}$ & $\bigcirc$ \\
$\begin{array}{l}\text { Lattice vibration } \\
\text { Atomic distribution } \\
\text { in real space }\end{array}$ & $\times$ & $\bigcirc$ & $\bigcirc$ & $\bigcirc$ \\
\hline $\begin{array}{l}\text { Anhamonicity of } \\
\text { potential energy }\end{array}$ & - & $\bigcirc^{* 1}$ & $\bigcirc^{* 2}$ & $\times$ \\
\hline $\begin{array}{l}\text { Number of variational } \\
\text { parameters associated } \\
\text { real space }\end{array}$ & - & $\times$ & $\times$ & $\times$ \\
\hdashline $\begin{array}{l}\text { Configurational } \\
\text { entropy }\end{array}$ & & many & a few & zero \\
\hline
\end{tabular}

is the number of $\sigma$ - atoms. In that circumstance, a temperature dependency of a free energy $\Psi$ is described as Fig. 6 for a given chemical potential. When we consider a local lattice relaxation, the free energy curves change the relative position as shown in Figs. 6 (a) and (b). In this manner, transformation temperature decreases.

Finally, we summarized various advantageous and disadvantageous features among present CVM, conventional CVM, CDCVM and Gaussian CVM in Table 4. (1) The conventional CVM is not formulated in continuous real space, while the present CVM is derived from the partition function in continuous real and momentum spaces. (2) Gaussian CVM considers only lattice vibration but not local lattice relaxation because it has not developed for multi component system. In the present CVM, on the other hand, the partition function is defined also in the discrete space of spin variables. Then, multi component system can be considered, and consequently the local lattice relaxation is taken into account. (3) One of the bottlenecks for an application of CDCVM to three-dimensional system is an intractable number of variational parameters. In the present CVM, local lattice relaxation energy and vibrational free energy are derived by integrating the partition function. Then, variational parameters associated with real space are not required. (4) The present CVM cannot consider atomic distribution and anharmonicity of potential energy.

\section{Conclusion}

We propose a modified CVM which explicitly consider lo- cal lattice relaxation and lattice vibration without variational parameters associated with real space.

By using present CVM, we calculated (1) standard free energy of pure $\mathrm{Cu}$ and $\mathrm{Au}$, and (2) enthalpy of mixing of random phase. Both calculations are in agreement with analytical solution and SQS. Finally, we estimated (3) free energy of mixing for $\mathrm{Cu}-\mathrm{Au}$ disordered phase at $800 \mathrm{~K}$. A contribution of local lattice relaxation to the free energy of mixing is not small in comparison with configurational entropy and formation energy for a fixed lattice.

\section{Acknowledgments}

This work was supported by Grant-in-Aid for JSPS Fellows 25-2373 and JSPS Grant-in-Aid for Scientific Research (B) 26289227 .

\section{REFERENCES}

1) R. Kikuchi: Phys. Rev. 81 (1951) 988-1003.

2) M. Asta: Acta Mater. 44 (1996) 4131-4136.

3) T. Mohri, D. de Fontaine and J. M. Sanchez: Metall. Trans. A 17 (1986) 189-194.

4) J. M. Sanchez, V. Pierron-Bohnes and F. Mejía-Lira: Phys. Rev. B 51 (1995) 3429-3437.

5) T. Mohri: J. Phase Equilib. Diff. 32 (2011) 537-542.

6) T. Mohri, K. Terakura, S. Takizawa and J. M. Sanchez: Acta Metall. Mater. 39 (1991) 493-501.

7) J. M. Sanchez, J. R. Barefoot, R. N. Jarrett and J. K. Tien: Acta Metall. 32 (1984) 1519-1525.

8) M. Enomoto and H. Harada: Metall. Trans. A 20 (1989) 649-664.

9) S. -H. Wei, A. A. Mbaye, L. G. Ferreira and Alex Zunger: Phys. Rev. B 36 (1987) 4163-4185.

10) J. C. Wang, M. Osawa, T. Yokokawa, H. Harada and M. Enomoto: Comp. Mater. Sci. 39 (2007) 871-879.

11) T. Mohri, M. Ohno and Y. Chen: J. Phase Equilib. Diff. 27 (2006) 4753.

12) D. de Fontaine, eds. by H. Ehrenreich, F. Seitz and T. David: Solid State Physics, (Academic Press, 1979) pp. 173-175.

13) R. Kikuchi and A. Beldjenna: Phys. A 182 (1992) 617-634.

14) A. Finel: Prog. Theor. Phys. Supplement 115 (1994) 59-68.

15) A. Finel and R. Tétot: Stability of Materials, eds. by A. Gonis, P. E. A. Turchi and J. Kudrnovský, (Springer, US, 1996) pp. 197-203.

16) R. Kikuchi: J. Phase Equilib. 19 (1998) 412-421.

17) G. C. Rota: Z. Wahrsch. Verw. Gebiete 2 (1964) 340-368.

18) J. H. van Lint and R. M. Wilson: A Course in Combinatorics, (Cambridge university press, New York, 2001) pp. 333-350.

19) G. An: J. Stat. Phys. 52 (1988) 727-734.

20) D. Ruelle: Commun. Math. Phys. 5 (1967) 324-329.

21) R. Hultgren, P. D. Desai, D. T. Hawkins, M. Gleiser and K. K. Kelley: Selected Values of the Thermodynamic Properties of the Elements, (American Society for Metals, Metals Park, Ohio, 1973) pp. 47-53.

22) R. Hultgren, P. D. Desai, D. T. Hawkins, M. Gleiser and K. K. Kelley: Selected Values of the Thermodynamic Properties of the Elements, (American Society for Metals, Metals Park, Ohio, 1973) pp. 149-159.

23) A. Zunger, S. H. Wei, L. G. Ferreira and J. E. Bernard: Phys. Rev. Lett. 65 (1990) 353-356.

24) R. Hultgren, P. D. Desai, D. T. Hawkins, M. Gleiser and K. K. Kelley: Selected Values of the Thermodynamic Properties of Binary Alloys, (American Society for Metals, Metals Park, Ohio, 1973) pp. 258-271. 